HEALTH-RELATED QUALITY OF LIFE IN CERVICAL CANCER SURVIVORS:

A POPULATION-BASED SURVEY

Running title: Long-term QoL after cervical cancer

Ida J. Korfage, $\mathrm{PhD}{ }^{1}$, senior researcher;

Marie-Louise Essink-Bot, MD PhD ${ }^{1,2}$, associate professor;

Floortje Mols, $\mathrm{PhD}^{3,4}$, post-doc researcher;

Lonneke van de Poll-Franse, $\mathrm{PhD}^{3,4}$, associate professor;

Roy Kruitwagen, MD PhD ${ }^{5,6}$, professor;

Marjolein van Ballegooijen, MD PhD ${ }^{1}$, assistant professor

Affiliations

1 Dept. of Public Health, Erasmus MC, University Medical Center Rotterdam, the Netherlands;

2 Dept. of Social Medicine, Academic Medical Centre, University of Amsterdam the Netherlands;

3 Center of Research on Psychology in Somatic Diseases (CoRPS), Tilburg University, the Netherlands;

4 Comprehensive Cancer Centre South (CCCS), Eindhoven Cancer Registry, the Netherlands.

5 TweeSteden Hospital, Department of Gynecology, Tilburg, the Netherlands,

6 Department of Obstetrics and Gynecology, Academic Hospital Maastricht, Maastricht, the Netherlands.

\title{
Details for correspondence
}

Erasmus MC,

Dept. of Public Health, Room Ae-108,

P.O. Box 2040,

3000 CA Rotterdam, The Netherlands

Tel. no.: $\quad+31107038460$

Fax no.: $\quad+31107038475$

E-mail: i.korfage@erasmusmc.nl

\section{Acknowledgment}

The study for the submitted manuscript was entirely funded by the Dutch Cancer Society (EMCR 2003-2775). The authors were completely independent from funders in conducting and writing this study. 


\section{Meeting presentations}

- Presented as " top ranked abstract" in the plenary session of the SMDM Europe conference, Engelberg, Switzerland, June 2008.

- Presented at the ISOQoL conference on Quality of Life, Toronto, Canada; October 2007. Abstract received the ISOQOL New Investigator Award.

\section{Conflicts of interests}

One of the authors, Marjolein van Ballegooijen, is principal investigator in a project on cost effectiveness of HPV vaccination, financed by GSK (a pharmaceutical company that produces HPV vaccines). 


\section{ABSTRACT}

Purpose In a population-based sample of cervical cancer survivors health-related quality of life (HRQoL) was assessed 2-10 years post-diagnosis.

Methods and Materials All patients diagnosed with cervical cancer in 1995-2003 in the Eindhoven region and alive after January 2006 were identified through the cancer registry. Generic HRQoL (SF-36, EQ-5D), cervical cancer-specific HRQoL (EORTC-QLQ-CX24), and anxiety (STAI-6) were assessed and compared with a reference population $(n=349)$. Data on tumor characteristics at diagnosis and disease progression were available.

Results A total of 291 women responded (69\%), mean age 53 (SD 13, range 31-88) years. Treatment had consisted of surgery $(n=195)$ or a combination of therapies $(n=75)$; one woman had not been treated. Of all women, $85 \%$ was clinically disease free, $2 \%$ had a recurrence/metastasis and in $13 \%$ this was unknown. After controlling for background characteristics (age, education, job and marital status, having children, and country of birth) generic HRQoL scale scores were similar to the reference population, except for worse mental health among the survivors. The most frequent symptoms were crampy pain in the abdomen or belly $(17 \%)$, urinary leakage (15\%), menopausal symptoms (18\%), and problems with sexual activity. Compared to the 6-10-year survivors, more sexual worry and worse body image were reported by the 2-5-year survivors. Compared to surgery only, especially primary radiotherapy was associated with an increased frequency of treatment-related side effects, also after controlling for age and disease stage at diagnosis and at follow-up.

Conclusions Most of the cervical cancer survivors were coping well, although their mental health was worse than in the reference population. Even after 2-10 years, radiotherapy was associated with an increased frequency of treatment-related side effects. 


\section{KEYWORDS:}

- Cervical cancer

- Health-related quality of life

- Long-term survivors

- Multicenter study

- Population-based study 


\section{Introduction}

Cervical cancer is an important cause of death in women, especially in developing countries. In

Europe, 60,000 women are diagnosed with cervical cancer annually. ${ }^{1}$ Due to improved therapies and early detection the number of women that survive cervical cancer has increased. ${ }^{2}$ These survivors have been diagnosed with a life-threatening disease and were confronted with their own mortality. They may still experience the physical side effects of cervical cancer therapy such as permanent consequences for sexual function and childbearing, as well as menopausal, urological and gastrointestinal symptoms. ${ }^{3}$ Survivors may also experience anxiety for recurrence of cervical cancer. Health-related quality of life (HRQoL), defined in this study as the quality of life relating to disease and/or treatment, has been described for the 1 to 5-6 years following treatment of cervical cancer. ${ }^{4-8}$ In, for example, the case of prostate cancer ${ }^{9}$ and in adult-onset cancers in general ${ }^{10}$ long-term cancer survivors showed a comparatively favorable HRQoL, while HRQoL was less favorable among nonHodgkin survivors. ${ }^{11}$ With the exception of a recent Korean study ${ }^{12}$, long-term HRQoL after diagnosis of cervical cancer, however, has only been described for small numbers of patients. ${ }^{13-15}$ The present study aimed to assess the generic and treatment-specific HRQoL of women who survived cervical cancer. For this, a population-based study was conducted among all women diagnosed with cervical cancer in the region of the Eindhoven Cancer Registry in the years 1995-2003. We hypothesized that HRQoL of survivors would be similar to the general population, that HRQoL would be better among long-term survivors than among short-term survivors, and that adjuvant radiotherapy after surgery would be associated with worse HRQoL than primary radiotherapy. 


\section{Methods}

\section{Setting and respondents}

The Eindhoven Cancer Registry (ECR) records data on all newly diagnosed cancer patients in the south of the Netherlands, an area with 2.3 million inhabitants, 17 hospital locations, and two large

radiotherapy institutes. ${ }^{16}$ In cooperation with the ECR and all gynecologic oncology departments in the region we conducted a population-based, cross-sectional survey. All women could be identified who were diagnosed with cervical cancer between 1 January 1995 and 31 December $2003(n=691)$. Women who had died could be excluded through linkage with the Central Bureau for Genealogy that collects data on all deceased Dutch citizens. We identified 444 women (64\%) who were still alive on 31 January 2006 (Figure 1); of this group, 8 women were not contacted as advised by their physician e.g. because of serious (mental) illness, and 15 addresses could not be verified. The remaining 421 survivors were sent a questionnaire by their (former) gynecologists. In the accompanying letter the women were asked to complete the questionnaire and were informed that, by returning the completed questionnaire, they consented to linkage of the questionnaire data with their disease history as registered by the ECR. Patients were assured that non-participation would have no consequences for their follow-up care or treatment. If the questionnaire was not returned within two months, a reminder letter with an additional copy of the questionnaire was sent. This study was part of a comprehensive evaluation of the Dutch cervical cancer screening program. The Ethics Committee of the Erasmus University Medical Center Rotterdam approved the research protocol.

\section{Respondents' characteristics}

The ECR routinely collects data on tumor characteristics, including date of diagnosis, tumor grade and stage (Tumor-Lymph Node-Metastasis [TNM] clinical classification), ${ }^{17}$ treatment, and on date of birth and co-morbidity at the time of diagnosis (a slightly adapted version of the Charlson co-morbidity index)..$^{18}$

Data on recurrences and/or metastasis were obtained through patient records of treating gynecologists and general physicians.

Information on marital status, education, co-morbidity, profession, and country of birth was obtained through the questionnaire. Educational level was classified as low (primary school or lower technical education), intermediate, or high (college/university degree). 
HRQoL of survivors compared with other groups

To assess non-response bias, respondents' data on tumor characteristics and primary therapies as registered by the ECR were compared with data of non-respondents. The latter group consisted of cervical cancer survivors who were alive on 31 January 2006, but did not complete the questionnaire, including women that had not been contacted because of ill health or unknown address.

To enable a better interpretation of respondents' generic HRQoL scores we included a reference group from the general population. Randomly selected women (aged 30-70 years, stratified in 10-year age groups) were sent a questionnaire through the regional screening organization in Maastricht. Overall response was $46 \%$. Data from the first 349 questionnaires that randomly became available after data input were included in the analyses for the current study.

The respondents' data were compared with two more data sets:

- For a comparison between survivors and non-survivors we present tumor characteristics and primary therapies of women who were diagnosed with cervical cancer in 1995-2003 and died before 31 January 2006;

- To enable a better interpretation of respondents' cervical cancer-specific HRQoL we use the validation study of the EORTC cervical cancer-specific module (QLQ-CX24). ${ }^{3}$

Finally, two comparisons were made within the respondents group, i.e. between those who survived 2-5 years vs. 6-10 years, and between those who received primary radiotherapy vs. surgery only vs. adjuvant radiotherapy after surgery.

\section{Questionnaire}

The questionnaire included validated measures on generic HRQoL, anxiety, cervical cancer-specific HRQoL and disease-related psychological distress.

Generic HRQoL was assessed by the 36-item Short-Form Health Survey (SF-36) and the EuroQol classification (EQ-5D). The SF-36 consists of 8 multi-item scales in the physical, social, and mental domain. Higher scale scores (0-100) indicate better functioning. ${ }^{19}$ The EQ-5D classification consists of 5 items (mobility, self-care, usual activities, pain/discomfort, and anxiety/ depression). Classification scores can be linked to a utility score with 0 indicating 'death' and 1 'full health'. ${ }^{20}$ The EQ-5D is complemented by a visual analog scale on current health, the Valuation of Own Health, which is 
anchored at the lower end ( 0 ) by 'worst imaginable health state' and at the upper end (100) by 'best imaginable health state'.

Anxiety was assessed by the STAI-6, a validated short version of the State Trait Anxiety Inventory containing 6 items on feeling at ease or upset. Higher scores (20-80) indicate higher levels of

anxiety. ${ }^{21,22}$ A STAI-State score of over 44 defines an individual as highly anxious. ${ }^{23}$

Disease-specific HRQoL was assessed through a recently validated cervical cancer-specific module (EORTC-QLQ-CX24), consisting of 3 multi-item scales on symptom experience, body image, and sexual/vaginal functioning and of 6 single-item scales. Higher scores (0-100) indicate worse functioning except for sexual activity and enjoyment. ${ }^{3}$

Disease-related psychological distress of having (had) cervical cancer was assessed by the Impact of Events Scale (IES). This measure includes a 7-item scale on intrusion referring to the degree the respondent was overwhelmed by thoughts and feelings about cervical cancer, and an 8-item avoidance scale referring to the tendency to keep off these thoughts and feelings. By summation of the intrusion (0-35) and the avoidance score (0-40) the total distress score is obtained, with higher scores indicating higher levels of distress. ${ }^{24}$

\section{Statistical analysis}

All statistical analyses were performed using SPSS for Windows, version 15.0.

Routinely collected data from the ECR on tumor characteristics enabled a comparison between respondents and non-respondents using Kruskall-Wallis non-parametric tests for continuous variables and Chi-square analyses for categorical variables. Procedures concerning imputation of missing responses in the SF-36 items were conducted according to the guidelines of the SF-36. ${ }^{19}$ General linear model (ANOVA) analyses were carried out to assess differences between respondents and the reference population.

The minimal important difference (MID), defined as the smallest change in a patient-reported outcome that is perceived by patients as beneficial or that would result in a change of treatment, was operationalized as a difference of at least half a standard deviation. ${ }^{25}$

General linear models were used to analyze the relation between time since diagnosis versus each of the 9 cervical cancer-specific subscales. General linear models that were used to analyze the relation between tumor characteristics and primary treatments versus HRQoL outcomes included age at 
survey and disease stage at diagnosis and at follow-up. In all analyses $p$-values less than 0.05 (referring to two-sided statistical tests) were considered significant. 


\section{Results}

\section{Respondents' characteristics}

Of the 421 cervical cancer survivors (response 69\%), 291 completed the questionnaire. The remaining 130 questionnaires were not completed due to wrong address $(n=11)$, patients' bad health $(n=2)$, lack of motivation $(n=2)$, death $(n=1)$, or unknown reasons $(n=114)$ (Figure 1). Compared to the reference group, survivors were older, had a lower education level, and lower rates of having paid jobs, having partners, and having children (Table 1).

\section{Clinical data}

The average age at diagnosis was 47 (range 25-83) years, average time since diagnosis was 6.6 (range 2-11) years (Table 2). Initial therapy consisted of surgery only in $67 \%$ of respondents, whereas $7 \%$ received radiotherapy only. The remaining patients were treated with a combination of surgery, radiotherapy, and/or chemotherapy, while one woman had not been treated. In April 2006, 85\% of survivors were clinically disease free, $2 \%$ had metastasis and/or local recurrence, and in $13 \%$ it was unknown whether recurrence had occurred. Compared to respondents, non-respondents had a significantly worse TNM stage at diagnosis and more local recurrence at follow-up. Deceased patients were older at diagnosis than survivors. Furthermore, tumor grade and TNM stage at diagnosis were worse than in survivors, and deceased patients had more often not been treated (Table 2).

\section{Health-related quality of life}

Survivors showed significantly lower SF-36 scores, indicating a worse quality of life, than the reference group (Table 3). However, after adjustment for significant differences in background characteristics (i.e. age at survey, education, job and marital status, having children or not, and country of birth) only mental health remained significantly worse in survivors. This difference between survivors and the reference group was larger than the MID (Table 3). Mean STAI-6 anxiety scores were worse in patients than in the reference group ( $p<0.001$ after correction for background variables). Among the survivors, $18 \%$ had a score above the cut-off value for being highly anxious, i.e. $>44,{ }^{23}$ vs. $15 \%$ in the reference sample.

In the present study, cervical cancer-specific HRQoL scores were lower (indicating a better functioning) than in the validation study of the EORTC cervical cancer-specific module (QLQ-CX24). ${ }^{3}$ 
However, more symptoms were reported in the current study, e.g. crampy pain in the abdomen or belly $(17 \%)$, urinary leakage (15\%), lymphedema (17\%), menopausal symptoms (18\%), and neuropathy $(15 \%)$.

\section{2-5- year vs. 6-10-year survivors}

Generic HRQoL was similar among the 2-5 and 6-10-year survivors (data not shown). STAI-6 scores were higher (indicating more anxiety) in the $2-5$-year survivors $(p=0.07)$. Cervical cancer-specific HRQoL differed only regarding body image $(p=0.004)$ and sexual worry $(p=0.04)$, which were worse in the 2-5-year survivors (Table 4). Univariate linear regression confirmed these findings.

Primary radiotherapy vs. adjuvant radiotherapy after surgery vs. surgery only

After correction for age at survey and disease stage at diagnosis and at follow-up, we compared three groups: primary radiotherapy (including chemoradiation), adjuvant radiotherapy after surgery, and surgery only. Generic HRQoL scores did not differ significantly between these groups (data not shown).

Cervical cancer-specific function was worse in women treated with radiotherapy, e.g. symptom experience $(p=0.006)$, sexual/vaginal functioning $(p<0.001$, but the numbers were small for this scale), and sexual worry $(p<0.001)$ (Table 5$)$, also for frequencies of reported symptoms per treatment group. Cervical cancer-specific HRQoL as reported after primary radiotherapy was worse than after adjuvant radiotherapy. The primary radiotherapy group consisted of women who received radiotherapy only $(n=20)$ and of women who received chemoradiation, i.e. a combination of radiotherapy and chemotherapy $(n=20)$. Comparing the treatment-specific HRQoL in these two groups, significant differences emerged for all but one of the EORTC QLQ-CX24 scale scores. The differences remained significant after controlling for differences in age at survey, and disease stage at diagnosis and at follow-up. Women treated by chemoradiation reported more symptom experience ( $p=0.001)$, body image ( $p=0.029)$, and sexual worry $(p=0.004)$. However, women treated by radiotherapy only reported more lymphedema $(p<0.001)$, peripheral neuropathy $(p=0.01)$, and menopausal symptoms $(p=0.01)$. 


\section{Discussion}

We assessed HRQoL in 291 cervical cancer survivors 2-10 years after diagnosis and treatment and found that the majority was doing well. However, cervical cancer-specific symptoms such as pain in the abdomen, urinary leakage, and menopausal symptoms were reported by several women and mental health was significantly worse than in the reference group. HRQoL scores of 2-5-year survivors were similar to those of the 6-10-year survivors except for more anxiety, worse body image and more sexual worry in the 2-5-year survival group.

After correction for age and disease stage at diagnosis and at follow-up, treatment-related HRQoL was significantly worse after radiotherapy than after surgery only. This was in line with our hypothesis and with the earlier reported trend that radiotherapy is generally more associated with reduced HRQoL than surgery or chemotherapy. ${ }^{2,26}$ Furthermore, more treatment-related symptoms were reported after primary radiotherapy than after adjuvant radiotherapy, a finding that did not correspond with our hypothesis. Half of the primary radiotherapy group had been treated with chemoradiation, the other half with radiotherapy only. We explored whether the high rate of treatment-related symptoms could be attributed to chemoradiation. Although we found significant differences between women treated by radiotherapy only vs. women treated by chemoradiation, one group was not overall worse than the other. We conclude that the high rate of treatment-related symptoms after primary radiotherapy can not be attributed to chemoradiation.

Cervical cancer survivors had a lower level of education and less often had paid jobs than the reference group, indicating a less favorable socioeconomic status (SES) in survivors. This finding corresponds with earlier data on the association between cervical cancer and low SES. In American studies the incidence of cervical cancer was repeatedly found to increase with socioeconomic deprivation among all included ethnic groups (Asian and Pacific Islander, black, Hispanic, and white). ${ }^{27-29}$

Our results confirm earlier findings in (mainly) small samples of long-term cervical cancer survivors. In a Swedish study among 46 cervical cancer survivors, 5-7 years after diagnosis, quality of life was satisfactory. ${ }^{7}$ Fifty-one US cervical cancer survivors (interviewed on average 8 years after diagnosis) were found to enjoy a good HRQoL, with physical, social, and emotional functioning comparable to or better than norm scores; however, in that study only $20 \%$ (51/254) of identified cervical cancer survivors completed the questionnaire. ${ }^{14}$ In another study reporting on 152 survivors of cervical or 
endometrial cancer, diagnosed and treated on average 11 years earlier, HRQoL was found to approximate that of healthy controls, although cervical cancer survivors reported more negative mood. ${ }^{13}$ Data of 74 patients ( 37 treated with surgery and 37 with radiotherapy), on average 7 years after diagnosis, suggested that cervical cancer survivors who were treated with surgery alone can expect an overall quality of life and sexual function not unlike that of peers without a history of cancer. ${ }^{15}$ A recent large study among Korean cervical cancer survivors $(n=860)$, diagnosed and treated 1.4-22 years earlier, showed that survivors reported more impaired social functioning, more bowel and urinary problems, and more menopausal symptoms than women from the general population ${ }^{12}$. As in our study, women treated by radiotherapy without surgery reported more peripheral neuropathy than others. Another corresponding finding was that women treated by radiotherapy experienced more sexual worry than women treated by surgery. Unfortunately, the response in this well-conducted latter study was only $12.4 \%$ (860 of 5943 potential subjects). The authors attribute the low response to the amount of time that passed since the cancer diagnosis, and the reluctance to provide personal information that is typical for Korean women ${ }^{12}$. In our study group, although generic HRQoL was relatively good several women reported treatmentrelated symptoms. Since the QLQ-CX24 module on cervical cancer-specific HRQoL has recently been published, we could compare our results with only two other studies: the EORTC validation study ${ }^{3}$ itself, and the recent Korean study on HRQoL in cervical cancer survivors. ${ }^{12}$ The QLQ-CX-24 scores of our sample were better than those of the EORTC validation study, which may be explained by the EORTC sample partly consisting of patients in the primary treatment phase versus survivors at 2-10 years after diagnosis in the current study. Except for menopausal symptoms, disease-specific function in our sample was also better than in the Korean sample, but we have no explanation for this difference.

It may seem strange that an increased prevalence of treatment-related symptoms was not reflected in lower generic HRQoL scores in cervical cancer survivors. A relatively good generic HRQoL in combination with treatment-related side effects has also repeatedly been reported by men with prostate cancer. ${ }^{9,} 30$ Although these men considered treatment-related side effects to be a problem, they did not take them into account when completing HRQoL measures because they did not consider them to be aspects of health. Furthermore prostate cancer patients accepted treatment-related side 
effects as an inevitable consequence of having been treated for cancer, a condition they perceived as life threatening. ${ }^{31}$ Similar processes may play a role in survivors of cervical cancer.

The strengths of our study are the large number of respondents ( $n=291$; response $69 \%$ ), the population-based unselected sample of survivors reflecting average care in the general population in the Netherlands, the cooperation of all departments of gynecologic oncology in the region, and the availability of registry data on tumor characteristics at diagnosis and of therapy. An additional strength is the use of standardized, valid measures, including the new cervical cancer-specific module of the EORTC, the QLQ-CX24. ${ }^{3}$ Anxiety and cervical cancer-specific symptoms (e.g. lymphedema and menopausal symptoms) were assessed together with generic HRQoL, as was recommended in a recent review on quality of life studies of long-term survivors of cervical cancer. ${ }^{2}$

Although the response rate of $69 \%$ in our study is reasonably good, our results can not necessarily be generalized to the whole group of cervical cancer survivors. Age, grade of differentiation at diagnosis, and time since diagnosis were similar in respondents and non-respondents. However, TNM stages were worse in the non-respondents and recurrences at follow-up were significantly more frequent in non-respondents than in respondents (4\% vs. $1 \%)$.

In $13 \%$ of respondents it was unknown whether metastases or recurrence had occurred, because their former gynecologists had not seen these women in recent years, and patient medical records contained no data on metastases or recurrence. The fact that a patient had not been seen by her treating physician may in itself be an indication that recurrence or metastasis probably had not occurred.

On average, patients who had died at the time of the survey were older at diagnosis and had worse tumor characteristics than survivors. Survival is known to be worse in older women with cervical cancer, which is caused in part by the worse tumor stage at diagnosis. ${ }^{32}$

The results of the present study may have implications for cost-effectiveness analyses of, for instance, programs for cervical cancer screening and HPV vaccinations. For such analyses HRQoL data are needed of women who survived cervical cancer, since the gains of preventing cervical cancer include the reduction of its incidence and thus a reduction of post-treatment phases in life. So far there is uncertainty with respect to the HRQoL associated with e.g. having invasive cancer. ${ }^{33} \mathrm{HRQ}$ oL effects as reported by survivors in this study can reduce this uncertainty. A clinical implication of the present study is that radiotherapy in general, and primary radiotherapy in particular, resulted in a higher 
frequency of treatment-related side effects than surgery, information that is useful for clinicians and patients when faced with the choice of cervical cancer therapy.

\section{ACKNOWLEDGMENTS}

The authors are grateful to the survivors and to the women from the reference group for participating in the study. We thank the gynecologists in the region of the Eindhoven Cancer Registry for their friendly cooperation. The authors are grateful to the Dutch Cancer Society for funding this research (EMCR 2003-2775). 


\section{REFERENCES}

1. Ferlay J, Bray F, Pisani P, Parkin DM. Globocan, 2004. Cancer incidence, mortality and prevalence worldwide.ed. Lyon: IARCPress, 2004.

2. Vistad I, Fossa SD, Dahl AA. A critical review of patient-rated quality of life studies of long-term survivors of cervical cancer. Gynecol Oncol 2006;102:563-72.

3. Greimel ER, Kuljanic Vlasic K, Waldenstrom AC, et al. The European Organization for Research and Treatment of Cancer (EORTC) Quality-of-Life questionnaire cervical cancer module: EORTC QLQ-CX24. Cancer 2006;107:1812-22.

4. Jensen PT, Groenvold M, Klee MC, et al. Longitudinal study of sexual function and vaginal changes after radiotherapy for cervical cancer. Int J Radiat Oncol Biol Phys 2003;56:937-49.

5. Bergmark K, Avall-Lundqvist E, Dickman PW, Henningsohn L, Steineck G. Vaginal changes and sexuality in women with a history of cervical cancer. $N$ Engl J Med 1999;340:1383-9.

6. Klee M, Thranov I, Machin D. Life after radiotherapy: the psychological and social effects experienced by women treated for advanced stages of cervical cancer. Gynecol Oncol 2000;76:5-13.

7. Li C, Samsioe G, losif C. Quality of life in long-term survivors of cervical cancer. Maturitas 1999;32:95-102.

8. Bye A, Trope C, Loge JH, Hjermstad M, Kaasa S. Health-related quality of life and occurrence of intestinal side effects after pelvic radiotherapy--evaluation of long-term effects of diagnosis and treatment. Acta Oncol 2000;39:173-80.

9. Korfage IJ, Essink-Bot ML, Borsboom GJ, et al. Five-year follow-up of health-related quality of life after primary treatment of localized prostate cancer. Int $J$ Cancer 2005;116:291-6.

10. Gotay CC, Muraoka MY. Quality of life in long-term survivors of adult-onset cancers. $J$ Natl Cancer Inst 1998;90:656-67. 
11. Mols F, Aaronson NK, Vingerhoets AJ, et al. Quality of life among long-term nonHodgkin lymphoma survivors: a population-based study. Cancer 2007;109:1659-67.

12. Park SY, Bae DS, Nam JH, et al. Quality of life and sexual problems in disease-free survivors of cervical cancer compared with the general population. Cancer 2007;110:2716-25.

13. Bradley S, Rose S, Lutgendorf S, Costanzo E, Anderson B. Quality of life and mental health in cervical and endometrial cancer survivors. Gynecol Oncol 2006;100:479-86.

14. Wenzel L, DeAlba I, Habbal R, et al. Quality of life in long-term cervical cancer survivors. Gynecol Oncol 2005;97:310-7.

15. Frumovitz M, Sun CC, Schover LR, et al. Quality of life and sexual functioning in cervical cancer survivors. J Clin Oncol 2005;23:7428-36.

16. Janssen-Heijnen M, Louwman W, Poll-Franse Lvd, Coebergh J, Results of 50 years cancer registry in the South of the Netherlands: 1955-2004 (in Dutch). Eindhoven Cancer Registry, 2005.

17. Hermanek P, Sobin LH. TNM Classification of Malignant Tumours. UICC International Union Against Cancers.ed. Berlin: Springer-Verlag, 1992.

18. Charlson ME, Pompei P, Ales KL, MacKenzie CR. A new method of classifying prognostic comorbidity in longitudinal studies: development and validation. J Chronic Dis 1987;40:373-83.

19. Ware JEJ, Snow KK, Kosinski M, Gandek BG. SF-36 health survey: manual and interpretation guideed.: Boston, MA : The Health Institute, New England Medical Center, 1993.

20. Dolan P. Modeling valuations for EuroQol health states. Med Care 1997;35:1095-108.

21. Marteau TM, Dormandy E, Michie S. A measure of informed choice. Health Expect 2001;4:99-108.

22. van der Bij AK, de Weerd S, Cikot RJ, Steegers EA, Braspenning JC. Validation of the dutch short form of the state scale of the Spielberger State-Trait Anxiety 
Inventory: considerations for usage in screening outcomes. Community Genet 2003;6:84-7.

23. Millar K, Jelicic M, Bonke B, Ashbury AJ. Assessment of preoperative anxiety: comparison of measures in patients awaiting surgery for breast cancer. Br J Anaesth 1995;74:180-3.

24. Nunnally J, Bernstein I. Psychometric theoryed. New York: McGraw-Hill, 1994.

25. Norman GR, Sloan JA, Wyrwich KW. Interpretation of changes in health-related quality of life: the remarkable universality of half a standard deviation. Med Care 2003;41:582-92.

26. Landoni F, Maneo A, Colombo A, et al. Randomised study of radical surgery versus radiotherapy for stage Ib-Ila cervical cancer. Lancet 1997;350:535-40.

27. Krieger N, Quesenberry C, Jr., Peng T, et al. Social class, race/ethnicity, and incidence of breast, cervix, colon, lung, and prostate cancer among Asian, Black, Hispanic, and White residents of the San Francisco Bay Area, 1988-92 (United States). Cancer Causes Control 1999;10:525-37.

28. Singh GK, Miller BA, Hankey BF, Edwards BK. Persistent area socioeconomic disparities in U.S. incidence of cervical cancer, mortality, stage, and survival, 19752000. Cancer 2004;101:1051-7.

29. Newmann SJ, Garner EO. Social inequities along the cervical cancer continuum: a structured review. Cancer Causes Control 2005;16:63-70.

30. Mols F, van de Poll-Franse LV, Vingerhoets AJ, et al. Long-term quality of life among Dutch prostate cancer survivors: results of a population-based study. Cancer 2006;107:2186-96.

31. Korfage IJ, Hak T, de Koning HJ, Essink-Bot ML. Patients' perceptions of the sideeffects of prostate cancer treatment--a qualitative interview study. Soc Sci Med 2006;63:911-9. 
32. de Rijke JM, van der Putten HW, Lutgens LC, et al. Age-specific differences in treatment and survival of patients with cervical cancer in the southeast of The Netherlands, 1986-1996. Eur J Cancer 2002;38:2041-7.

33. Goldie SJ, Kim JJ, Wright TC. Cost-effectiveness of human papillomavirus DNA testing for cervical cancer screening in women aged 30 years or more. Obstet Gynecol 2004;103:619-31. 
Figure 1. Flow chart of the study population

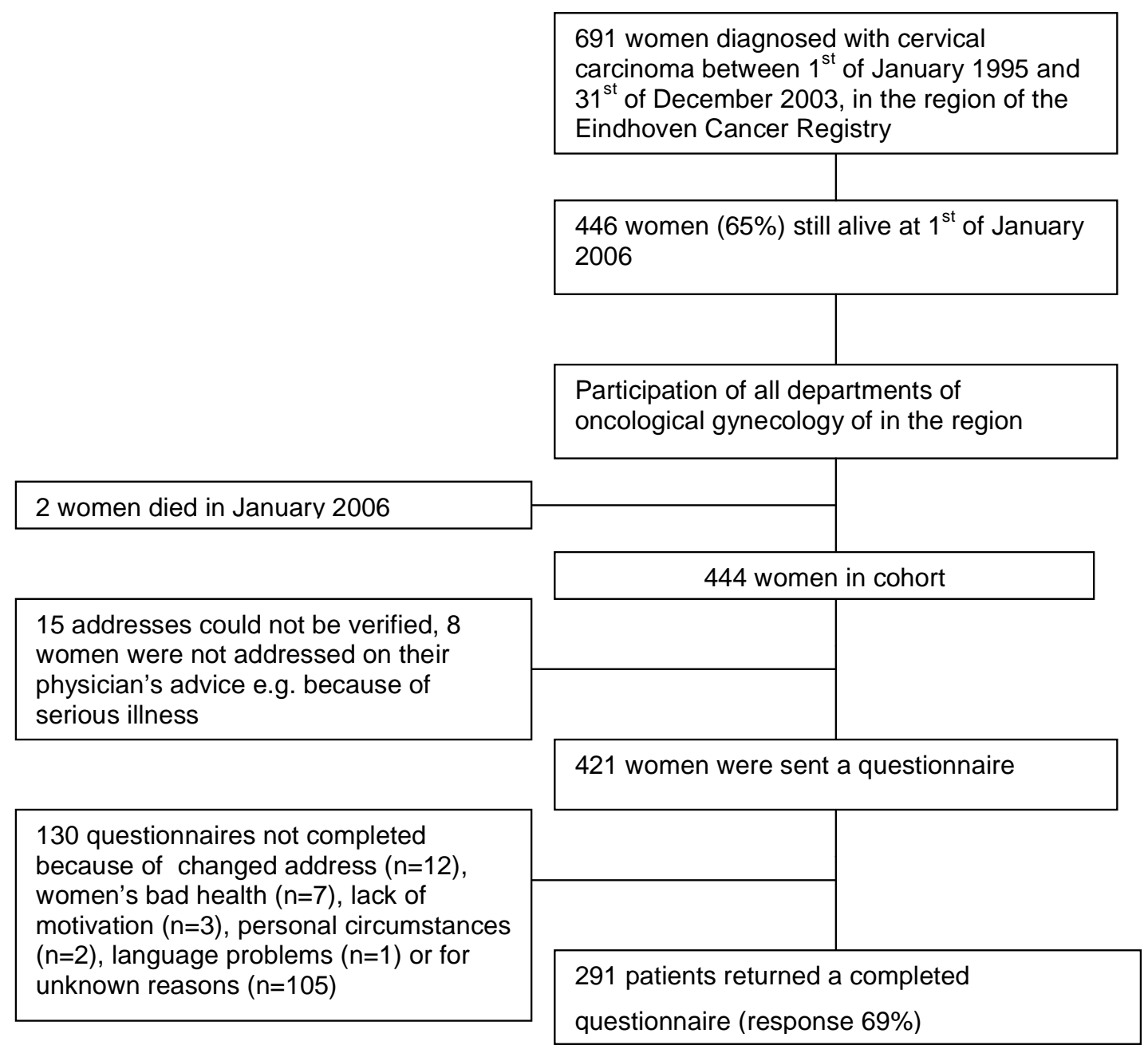


Table 1. Background variables of survivors, i.e. women diagnosed with cervical cancer in the years 1995 - 2004 and alive at $1^{\text {st }}$ of January 2006, and a reference group of women without cervical cancer randomly selected from the general population $(n, \%)$. The $p$-values indicate the significance level of differences between these groups.

\begin{tabular}{|c|c|c|c|c|c|}
\hline & \multicolumn{2}{|c|}{$\begin{array}{c}\text { Survivors } \\
n=291\end{array}$} & \multicolumn{2}{|c|}{$\begin{array}{l}\text { Reference group } \\
\quad \mathrm{n}=349\end{array}$} & $p$-value \\
\hline Age at time of the survey (years) & & & & & 0.015 \\
\hline Average (SD) & \multicolumn{2}{|c|}{$52.9(13.8)$} & \multicolumn{2}{|c|}{$50.5(10.8)$} & \\
\hline Range & \multicolumn{2}{|c|}{$31-88$} & \multicolumn{2}{|c|}{$27-70$} & \\
\hline Education & & & & & 0.003 \\
\hline Low education & 115 & $44 \%$ & 103 & $32 \%$ & \\
\hline Medium & 118 & $45 \%$ & 159 & $49 \%$ & \\
\hline High & 29 & $11 \%$ & 60 & $19 \%$ & \\
\hline Job status & & & & & 0.001 \\
\hline Paid job & 96 & $41 \%$ & 183 & $58 \%$ & \\
\hline Housewife/unpaid job & 83 & $37 \%$ & 83 & $26 \%$ & \\
\hline No job & 28 & $12 \%$ & 20 & $6 \%$ & \\
\hline Retired & 22 & $10 \%$ & 29 & $9 \%$ & \\
\hline Marital status & & & & & 0.001 \\
\hline Married/cohabiting & 188 & $66 \%$ & 273 & $78 \%$ & \\
\hline Partner, but living alone & 16 & $6 \%$ & 8 & $2 \%$ & \\
\hline No partner & 80 & $29 \%$ & 68 & $20 \%$ & \\
\hline \multicolumn{6}{|l|}{ Children } \\
\hline No & 61 & $22 \%$ & 48 & $15 \%$ & 0.03 \\
\hline Yes & 224 & $78 \%$ & 279 & $85 \%$ & \\
\hline Average no. of children, range & \multicolumn{2}{|c|}{$2,1-11$} & \multicolumn{2}{|c|}{$2,1-7$} & 0.013 \\
\hline \multicolumn{6}{|l|}{ Country of birth } \\
\hline the Netherlands & 261 & $92 \%$ & 327 & $99 \%$ & $<0.001$ \\
\hline \multicolumn{6}{|l|}{ Country of birth of parents } \\
\hline Father born in the Netherlands & 253 & $92 \%$ & 316 & $99 \%$ & $<0.001$ \\
\hline Mother born in the Netherlands & 248 & $89 \%$ & 311 & $99 \%$ & $<0.001$ \\
\hline Both parents of non-Dutch descent & 20 & $7 \%$ & 2 & $1 \%$ & \\
\hline
\end{tabular}


Table 2. Clinical data of all women in the Southeast of the Netherlands who were diagnosed with cervical cancer in the years 1995-2004 (n, \%), p-values relate to differences between respondents and non-respondents.

\begin{tabular}{ccccc}
\hline & $\begin{array}{c}\text { Respondents } \\
(\mathrm{n}=291)\end{array}$ & $\begin{array}{c}\text { Non- } \\
\text { respondents* } \\
(\mathrm{n}=153)\end{array}$ & $p$-value & $\begin{array}{c}\text { Deceased } \\
\text { patients** } \\
(\mathrm{n}=247)\end{array}$ \\
\hline $\begin{array}{c}\text { Age at diagnosis (years) } \\
\text { Average (SD) } \\
\text { Range }\end{array}$ & $47(14)$ & $48(14)$ & $\mathbf{0 . 2 8}$ & $61(18)$ \\
& $25-83$ & $15-81$ & & $24-100$
\end{tabular}

Years since diagnosis

$\begin{array}{lccc}\text { Average (SD) } & 6.6(2.6) & 6.8(2.6) & \mathbf{0 . 2 9} \\ \text { Range } & 2.0-11.0 & 2-11 & \end{array}$

\section{Grade at diagnosis}

1 Well differentiated

2 Moderately differentiated

3 Poorly differentiated

4 Undifferentiated

Missing

TNM stage ${ }^{\#}$ at diagnosis (\%)

0
1
2
3
4
missing

\begin{tabular}{rccr}
26 & $14 \%$ & 10 & $11 \%$ \\
83 & $46 \%$ & 44 & $48 \%$ \\
68 & $38 \%$ & 38 & $41 \%$ \\
3 & $2 \%$ & \multicolumn{2}{c}{-} \\
110 & & 61 &
\end{tabular}

$\begin{array}{rcrr}1 & - & 1 & 1 \% \\ 204 & 71 \% & 91 & 62 \% \\ 43 & 15 \% & 27 & 18 \% \\ 35 & 12 \% & 24 & 16 \% \\ 4 & 1 \% & 4 & 3 \% \\ 4 & & 6 & \end{array}$

0.51

$\begin{array}{cc}18 & 10 \% \\ 71 & 38 \% \\ 90 & 48 \% \\ 9 & 5 \% \\ 59 & \end{array}$

0.37

$\begin{array}{ll}51 & 22 \% \\ 53 & 23 \% \\ 67 & 28 \% \\ 65 & 28 \% \\ 11 & \end{array}$

Initial therapy (may contain several treatments per woman)

\begin{tabular}{lrrrrrrr} 
Surgery & 250 & $86 \%$ & 121 & $79 \%$ & $\mathbf{0 . 0 7}$ & 84 & $34 \%$ \\
Radiotherapy & 91 & $31 \%$ & 57 & $37 \%$ & $\mathbf{0 . 2 0}$ & 162 & $66 \%$ \\
$\quad$ External beam radiotherapy & 83 & $29 \%$ & 55 & $36 \%$ & $\mathbf{0 . 1 1}$ & 155 & $63 \%$ \\
$\quad$ Internal radiotherapy & 61 & $21 \%$ & 37 & $24 \%$ & $\mathbf{0 . 4 4}$ & 84 & $34 \%$ \\
$\quad$ Unknown form & 5 & $2 \%$ & 3 & $2 \%$ & $\mathbf{0 . 8 6}$ & 8 & $3 \%$ \\
Chemotherapy & 29 & $10 \%$ & 13 & $9 \%$ & $\mathbf{0 . 6 2}$ & 37 & $15 \%$ \\
No therapy & 1 & $0 \%$ & 1 & $1 \%$ & $\mathbf{0 . 6 4}$ & 34 & $14 \%$ \\
\hline
\end{tabular}




\section{Therapy-combinations}

Surgery only

Surgery and radiotherapy

Surgery, radiotherapy and

chemotherapy

Surgery and chemotherapy

Radiotherapy only

Radiotherapy and chemotherapy

Chemotherapy only

No therapy

Metastases at follow-up (April 2006)

Yes
Unknown
No

No

$\begin{array}{rc}195 & 70 \% \\ 46 & 16 \% \\ 5 & 2 \%\end{array}$

$4 \quad 1 \%$

$20 \quad 7 \%$

$20 \quad 7 \%$

-

$10 \%$

$\begin{array}{rr}93 & 61 \% \\ 25 & 16 \% \\ 3 & 2 \%\end{array}$

$\begin{array}{rc}20 & 13 \% \\ 9 & 6 \% \\ 1 & 1 \% \\ 1 & 1 \%\end{array}$

0.07

$\begin{array}{rrrr}4 & 1 \% & 2 & 1 \% \\ 38 & 13 \% & 33 & 22 \% \\ 249 & 86 \% & 118 & 77 \%\end{array}$

\subsection{3}

$\begin{array}{rr}36 & 15 \% \\ 41 & 17 \% \\ 6 & 3 \% \\ & \\ 1 & 0 \%-- \\ 92 & 38 \% \\ 23 & 10 \% \\ 7 & 3 \% \\ 34 & 14 \%\end{array}$

Recurrences at follow-up (April 2006)

$\begin{array}{lrccc}\text { Yes } & 2 & 1 \% & 6 & 4 \% \\ \text { Unknown } & 37 & 13 \% & 34 & 22 \% \\ \text { No } & 252 & 87 \% & 113 & 74 \%\end{array}$

$\left.{ }^{*}\right)$ non-respondents are women who did not return the questionnaire $(n=130)$ or who were not addressed because lost to follow-up ( $n=15)$ or too ill according to their physician $(n=8)$.

$\left.{ }^{* *}\right)$ deceased women were diagnosed with cervical cancer in 1995-2003 and were not alive anymore at January 31st of January 2006.

\# TNM stage (Tumor-Node-Metastasis clinical classification) is identical to FIGO stage. 
Table 3. Observed generic quality of life scores (SD) of survivors, i.e. women diagnosed with cervical cancer in the years 1995 - 2004, and the reference group. The first column of $p$-values indicates the significance level of differences in observed scores, the second column of $p$-values indicates the significance level of differences in scores after controlling for differences in age at survey, education, job and marital status, having children or not, and country of birth.

\begin{tabular}{|c|c|c|c|c|}
\hline & $\begin{array}{l}\text { Survivors } \\
n=291\end{array}$ & $\begin{array}{l}\text { Reference group } \\
\qquad \mathrm{n}=349\end{array}$ & $p$-value & $\begin{array}{c}p \text {-value after controlling } \\
\text { for differences in } \\
\text { background variables }\end{array}$ \\
\hline \multicolumn{5}{|l|}{ SF-36 (0-100) } \\
\hline Physical function & $79.1(26.1)$ & $86.1(18.6)$ & $<0.001$ & 0.15 \\
\hline Role-physical & $73.0(39.6)$ & $82.7(32.4)$ & 0.001 & 0.38 \\
\hline Bodily pain & $83.4(23.0)$ & $85.1(19.2)$ & 0.33 & 0.34 \\
\hline General health & $68.2(22.6)$ & $71.3(19.4)$ & 0.07 & 0.80 \\
\hline Vitality & $63.9(21.1)$ & $68.0(18.5)$ & 0.011 & 0.35 \\
\hline Social functioning & $82.0(22.3)$ & $87.7(17.8)$ & 0.001 & 0.18 \\
\hline Role emotional & $80.8(37.2)$ & $88.2(29.5)$ & 0.007 & 0.50 \\
\hline Mental health & $67.4(13.1)$ & $79.3(17.1)$ & $<0.001$ & $<0.001 *$ \\
\hline \multicolumn{5}{|l|}{ EuroQol (0-100) } \\
\hline EQ-5D utility score & $81.4(24.4)$ & $87.5(19.9)$ & 0.001 & 0.38 \\
\hline Valuation of own health & $76.4(19.6)$ & $79.8(12.9)$ & 0.009 & 0.88 \\
\hline Stai-6 (20-80) & $36.0(11.2)$ & 37.7 (7.2) & 0.025 & $<0.001$ \\
\hline
\end{tabular}

$\mathrm{SD}=$ standard deviation.

Higher scores indicate better functioning, except for Stai-6.

*) Differences exceed the Minimal Important Difference (MID). 
Table 4. Disease-specific quality of life scores (SD) of women diagnosed with cervical cancer in the years 1995 - 2004 and alive at January $1^{\text {st }}, 2006$, for the entire group of survivors who responded, 2-5 yrs survivors, and 6-10 yrs survivors respectively. P-values refer to differences between the last 2 groups.

\begin{tabular}{|c|c|c|c|c|}
\hline & $\begin{array}{l}\text { All survivors } \\
n=291\end{array}$ & $\begin{array}{c}2-5 \text { yrs survivors } \\
n=126\end{array}$ & $\begin{array}{c}6-10 \text { yrs survivors } \\
n=165\end{array}$ & $p$-value \\
\hline \multicolumn{5}{|l|}{ Age } \\
\hline Mean (Standard Deviation) & $52.9(13.8)$ & $52.4(13.9)$ & $53.4(13.7)$ & 0.61 \\
\hline \multicolumn{5}{|l|}{ EORTC QLQ-CX24 (0-100) } \\
\hline \multicolumn{5}{|l|}{ Multi-item scales } \\
\hline Symptom Experience & $15.1(14.1)$ & $16.7(14.6)$ & $14.0(13.7)$ & 0.12 \\
\hline Body Image & $15.9(23.9)$ & $20.7(26.4)$ & $12.2(21.2)$ & 0.004 \\
\hline Sexual/vaginal functioning & $16.2(22.3)$ & $17.6(24.4)$ & $15.0(20.5)$ & 0.49 \\
\hline \multicolumn{5}{|l|}{ Single-item scales } \\
\hline Lymphoedema & $18.5(31.1)$ & $15.2(28.3)$ & $21.1(32.9)$ & 0.13 \\
\hline Peripheral Neuropathy & $18.7(28.3)$ & $20.4(31.3)$ & $17.4(26.0)$ & 0.41 \\
\hline Menopausal Symptoms & $24.1(31.3)$ & $25.7(34.9)$ & $22.8(28.3)$ & 0.45 \\
\hline Sexual Worry & $13.8(27.5)$ & $17.9(31.7)$ & $10.7(23.4)$ & 0.04 \\
\hline Sexual Activity & $26.9(26.4)$ & $27.6(25.1)$ & $26.4(27.4)$ & 0.73 \\
\hline Sexual Enjoyment & $58.3(30.1)$ & $56.2(30.8)$ & $60.1(29.5)$ & 0.44 \\
\hline \multicolumn{5}{|l|}{ Impact of Event Scale } \\
\hline Intrusion score (0-35) & $6.3(7.7)$ & $6.9(8.2)$ & $5.8(7.3)$ & 0.25 \\
\hline Avoidance score (0-40) & $7.1(8.5)$ & $7.9(8.5)$ & $6.5(8.5)$ & 0.19 \\
\hline Total distress score (0-75) & $13.4(15.0)$ & $14.8(15.6)$ & $12.3(14.4)$ & 0.17 \\
\hline
\end{tabular}

Higher scores indicate worse functioning, except for Sexual Activity and Sexual Enjoyment. 
Table 5. Mean values (SD) of cervical cancer specific quality of life (assessed through EORTC QLQ-CX24) and frequency of treatment related symptoms in \% of women that reported to have experienced them 'quite a bit' or 'rather much' during the week before completion of the survey.

\begin{tabular}{|c|c|c|c|c|}
\hline Therapy & $\begin{array}{c}\text { Primary } \\
\text { radiotherapy }{ }^{\#} \\
\mathrm{n}=40\end{array}$ & $\begin{array}{c}\text { Surgery } \\
\text { only } \\
n=195\end{array}$ & $\begin{array}{c}\text { Adjuvant radiotherapy } \\
\text { after surgery } \\
n=46\end{array}$ & $p$-value \\
\hline \multicolumn{5}{|l|}{ Age } \\
\hline Mean (SD) & $63.6(15.9)$ & $49.6(11.8)$ & $58.7(14.9)$ & $<0.001$ \\
\hline Symptom Experience (0-100) & $18.5(16.2)$ & $12.9(12.8)$ & $19.4(13.9)$ & 0.006 \\
\hline Cramps in abdomen & $24 \%$ & $14 \%$ & $21 \%$ & \\
\hline Difficulty in controlling bowels & $21 \%$ & $6 \%$ & $21 \%$ & \\
\hline Blood in stools & $3 \%$ & $1 \%$ & $2 \%$ & \\
\hline Pass water/urine frequently & $42 \%$ & $24 \%$ & $45 \%$ & \\
\hline Pain or burning feeling when urinating & $8 \%$ & $3 \%$ & $7 \%$ & \\
\hline Leaking of urine & $19 \%$ & $10 \%$ & $26 \%$ & \\
\hline Difficulty emptying bladder & $6 \%$ & $10 \%$ & $11 \%$ & \\
\hline Pain in lower back & $22 \%$ & $24 \%$ & $19 \%$ & \\
\hline Irritation/soreness in vagina or vulva & $5 \%$ & $5 \%$ & $7 \%$ & \\
\hline Discharge from vagina & $5 \%$ & $7 \%$ & $5 \%$ & \\
\hline Abnormal bleeding from vagina & $3 \%$ & $1 \%$ & $7 \%$ & \\
\hline Body Image (0-100) & $26.3(30.6)$ & $11.2(19.4)$ & $23.3(28.2)$ & 0.202 \\
\hline Felt physically less attractive & $24 \%$ & $5 \%$ & $18 \%$ & \\
\hline Felt less feminine & $18 \%$ & $4 \%$ & $16 \%$ & \\
\hline Felt dissatisfied with body & $34 \%$ & $13 \%$ & $25 \%$ & \\
\hline Lymphoedema (0-100) & $11.7(27.5)$ & $15.8(28.8)$ & $34.9(37.1)$ & 0.005 \\
\hline Swelling in one or both legs & $11 \%$ & $13 \%$ & $34 \%$ & \\
\hline
\end{tabular}


Peripheral Neuropathy (0-100)

Tingling or numbness in hands or feet

Menopausal Symptoms (0-100)

Hot flushes and/or sweats

Sexual Worry (0-100)

Worried that sex would be painful

Sexual Activity (0-100) *

Sexually active in past 4 weeks?

Quite a bit or rather much

A little

IF SEXUALLY ACTIVE:

Sexual/vaginal functioning (0-100)

Vagina felt dry during sexual activity (n, \%)

Inconvenience because vagina felt short (n, \%)

Inconvenience because vagina felt tight (n, \%)

Pain during sexual intercourse/activity (n, \%)

Sexual Enjoyment (0-100) *

Sexual activity enjoyable?
$24 \%$

$29.7(37.5)$

$24 \%$

$25.3(39.1)$

$7.8(18.3)$

$27 \%$

$13.3(20.1)$

$31.4(27.5)$

$6 \%$

$29 \%$

$\mathrm{n}=11$

$\mathrm{n}=115$

47.7 (30.3)

$11.0(16.6)$

5, $46 \%$

$15,13 \%$

$22.9(25.3)$

$6,55 \%$

7, $64 \%$

$4,36 \%$

36.4 (23.4)

$63.3(29.0)$

$3,27 \%$

$82,70 \%$

0.06
$50(31.5)$

$4,4 \%$

5, $4 \%$

$3,3 \%$

$10,50 \%$

$2,10 \%$

$3,15 \%$

4, $20 \%$ $n=20$

$<0.001$

0.40 $<0.001$

0.12

5, $25 \%$

\#) Primary radiotherapy: women treated by radiotherapy only or by radiotherapy and chemotherapy.

\#\#) p-value after correction for age at survey, and disease stage at diagnosis and at follow-up.

Higher scale scores indicate worse functioning, except for Sexual Activity and Sexual Enjoyment.

*) These scales do not represent 'symptoms'. 